\title{
DELAMINATION PROPERTIES OF THE HUMAN THORACIC ARTERIAL WALL WITH EARLY STAGE OF ATHEROSCLEROSIS LESIONS
}

\author{
MARTA KOZUŃ \\ Wroclaw University of Technology, Faculty of Mechanical Engineering, Wroctaw, Poland \\ e-mail:marta.kozun@pwr.edu.pl
}

\begin{abstract}
The aim of this work is to determine mechanical properties of interfaces between layers of the human thoracic aortic wall with early stages of atherosclerosis lesions. Circumferential $(n=48)$ and axial $(n=15)$ specimens have been prepared and the mechanical properties of the interfaces between the layers have been determined on the basis of the peeling test. The results show that the mechanical and dissection properties of the interfaces between the layers depend on the direction of the tests. The results confirm that the early stage of atherosclerosis does not affect the mechanical parameters of the layer interfaces and does not affect resistance of the vessel wall to delamination.
\end{abstract}

Keywords: peeling test, human arterial wall, thoracic artery, mechanical properties, atherosclerosis

\section{Introduction}

Human arterial wall is a three-layer (the intima, media, and adventitia) laminate reinforced with long fibres (collagen and elastin fibres), which play a key role in the transfer of mechanical stress. Collagen and elastin fibres are held together by the extracellular matrix.

Each layer of the arterial wall is characterized by a different structure, orientation of collagen and elastic fibres, mechanical properties, and function performed in the vessel wall (Holzapfel et al., 2004). The intimal layer is built mostly of connective tissue (Shekhonin et al., 1985), and in young and healthy people it is not involved in the transfer of mechanical stress. In the physiological range of blood pressure. this process is carried out by the media (collagen fibres, elastin fibres, and smooth muscle cells, see Kobielarz et al. (2013), Gąsior-Głogowska et al. (2011), Schriefl et al. (2012). The collagen fibres in this layer are arranged circumferentially (Schriefl et al., 2012). In the case of arterial hypertension, the process of transfer of stress also involves the adventitia (collagen fibres) (Shekhonin et al., 1985), which protects the vessel against overstretching and rupture (Holzapfel 2008; Schulze-Bauer et al., 2001). In the adventitia, collagen fibres are dispersed, and only individual fibres are arranged circumferentially (Schriefl et al., 2012). The cohesive composite structure of the human thoracic aortic wall ensures its high mechanical strength and determines its proper functioning, i.e. transfer of mechanical stress resulting from blood pressure and the ability to deform reversibly (Sommer et al., 2010).

Delamination of the vessel wall may occur spontaneously or as a result of trauma. Spontaneous dissection occurs in 5-30 cases per million people/year and depends on a number of factors (hypertension, atherosclerosis, aortic dilatation, and Marfans and Ehlers-Danlos syndromes (Tong et al., 2011). This kind of dissection is usually the result of structural remodelling of the aortic wall, which progresses along with the development of vascular diseases, including atherosclerosis. Degenerative changes that develop along with the progression of atherosclerosis, in particular the formation of atherosclerotic plaque (Kot et al., 2011), lead to changes in the mechanical properties of the individual layers of the aortic wall (Teng et al., 2009; Weisbecker 
et al., 2012) and affect the adhesion between them (Karimi et al., 2013). This leads to a loss of integrity of the vessel wall and, consequently, to its delamination. Aortic dissection of traumatic origin is, in most cases, the result of a diagnostic or therapeutic procedure performed on the vascular system, for example insertion of a stent graft or aortic stents. This treatment causes denudation of the endothelium, disruption of the intima and the atherosclerotic plaque with frequent separation from or dissection of the media, and overstretching of non-diseased portions of the arterial wall (Sommer et al., 2008). These intimal defects can cause an imbalance of distribution of mechanical stress on the arterial wall and may be the trigger for propagation of the aortic dissection. Due to its dynamic progression, diagnostic difficulties, and high mortality, aortic dissection is a difficult clinical issue. According to statistics, in the first 48 hours of the onset of dissection, the mortality rate is $1 \%$ per hour among untreated patients, about $16-20 \%$ of patients survive 14 days, and only 5\% survive 12 months (Szpakowski et al., 2006). Although arterial dissection is a frequently occurring phenomenon, the underlying biomechanical properties of arterial dissection remain largely unclear.

The research carried out in recent years on cardiovascular biomechanics has concerned mainly the analysis of the impact of pressure on the vessel wall. The interaction between the aortic wall and blood is also modelled, and the mechanical properties of the vessel wall and its layers are determined. The mechanical properties (maximum strain, tensile strength, and Young's modulus) are determined mostly by a uniaxial tension test (Kobielarz and Jankowski, 2013; Vorp et al., 1996, 2003) in radial, circumferential, and axial directions (Sommer et al., 2008). The results of experimental research obtained in this regard are important for description of the mechanism of vessel wall destruction but they are insufficient to determine pathogenesis of its dissection. Delamination of the vessel wall as a three-layer laminate reinforced with collagen and elastin fibres was considered by Sommer et al. (2008) and Tong et al. (2011). The authors proposed a new experimental method (peeling test), which allows them to determine the mechanical parameters of the interfaces between the layers of the vessel wall and the energy required to initiate and propagate dissection. Sommer et al. (2008) investigated the human abdominal aorta. Only the medial layer of the vessel wall was assessed in circumferential and axial directions. Tong et al. (2011) conducted a peeling test of human carotid bifurcations. The authors determined the mechanical properties and the energy dissipated during the dissection of adventitia-media and media-intima composites in circumferential and axial directions. Research conducted by Sommer et al. (2008) and Tong et al. (2011) focused on normal specimens while the problem of dissection concerned vessel walls with lesions arising due to development of atherosclerosis. Therefore, in the opinion of the author, the results of the studies on vessel wall delamination conducted so far have not fully explained the mechanism of this process.

Based on the above, the aim of the study is to determine the mechanical parameters of the interfaces between the layers of the human thoracic aortic wall in the early stage of atherosclerosis, including energy dissipated during the process of delamination (cracking) of the aortic wall. The obtained results may serve as the basis for the development of constitutive models of the arterial wall. None of the currently existing models takes into account the problem of delamination of the arterial wall, which is a major limitation because many diagnostic procedures as well as flow-related issues are based on the aforementioned models.

\section{Material and method}

The study has been conducted on human thoracic aortas collected at autopsy within 24 hours of death. A total of 14 specimens qualified for the study (male; age range: $29 \pm 12$ ) showing early atherosclerotic lesions (stage II of the development of atherosclerosis according to the classification proposed by Stary $(2000,2004)$. Each specimen was dissected parallel to the long 
axis of the vessel with a pair of surgical scissors. Next, a blanking tool was used on each aorta to punch out flat rectangular specimens with fixed dimensions of $5 \mathrm{~mm}$ (width) by $25 \mathrm{~mm}$ (length). The specimens were cut out in two orthogonal directions: in the circumferential direction $(C)$ $(n=48)$ and in the axial direction $(A)(n=15)$ (Fig. 1).

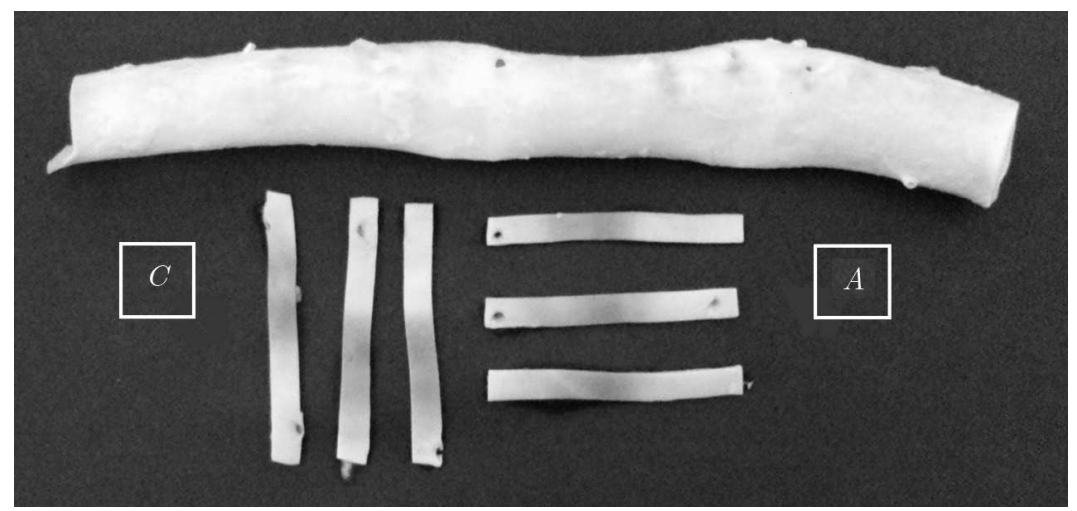

Fig. 1. Directions of specimen preparation: axial $(A)$ and circumferential $(C)$

The specimens were initially dissected over a length of about $5 \mathrm{~mm}$. This way two "tongues" were obtained for mounting the specimen into the testing machine (Fig. 2). Dissection was introduced between the following interfaces:

- adventitia $(A) \rightarrow$ media $(M)+$ intima $(I)$ (interface 1)

- $\operatorname{adventitia}(A)+$ media $(M) \rightarrow$ intima $(I)$ (interface 2)

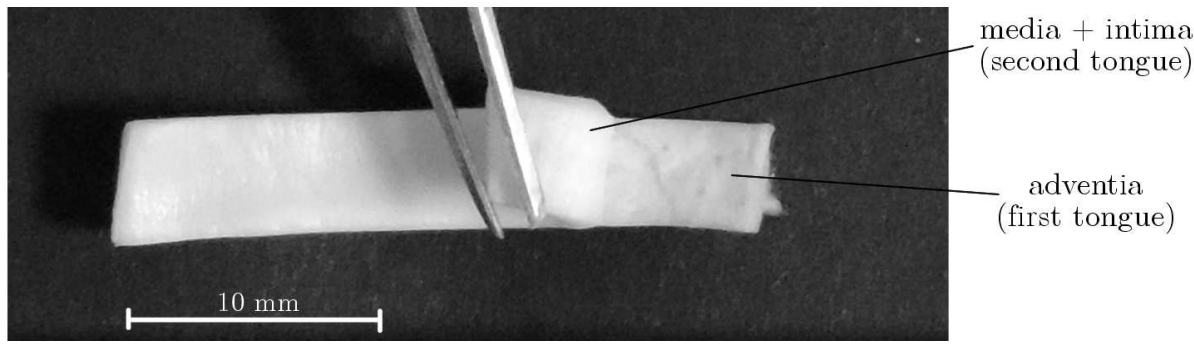

Fig. 2. Preparation of the material for tests of the mechanical properties - initial dissection of the axial specimen of the vessel wall. Width was measured for each specimen

The number of specimens prepared for tests is presented in Table 1. Until the performance of the tests, the specimens were stored in saline solution $(0.9 \% \mathrm{NaCl})$ at room temperature (15 minutes).

Table 1. The number of specimens prepared for testing of the mechanical properties

\begin{tabular}{|c|c|}
\hline Type of interface & Number of specimens \\
\hline \hline \multicolumn{2}{|c|}{ Circumferential direction } \\
\hline interface 1 & 26 \\
\hline interface 2 & 22 \\
\hline \multicolumn{2}{|c|}{ Axial direction } \\
\hline interface 1 & 7 \\
\hline interface 2 & 8 \\
\hline
\end{tabular}

Both sides of the two "tongues" of each specimen were fixed to two grips of the testing machine. The study used the material testing system (MTS) Synergie 100 machine. The mechanical properties of the interfaces between vessel wall layers were determined on the basis of the 
research methodology proposed by Sommer in 2008, the so-called peeling test (Sommer et al., 2008,2010 ). The load was applied perpendicular to the specimen dissection plane (T-peel test configuration) (Fig. 3). The test was conducted at a constant crosshead speed of $2 \mathrm{~mm} / \mathrm{min}$ in two directions: axial and circumferential. The testing was carried out under repeatable conditions at a constant ambient temperature. During the test, changes were recorded in the value of force $(F)$ as a function of displacement $(d)$ in the direction of the applied load.
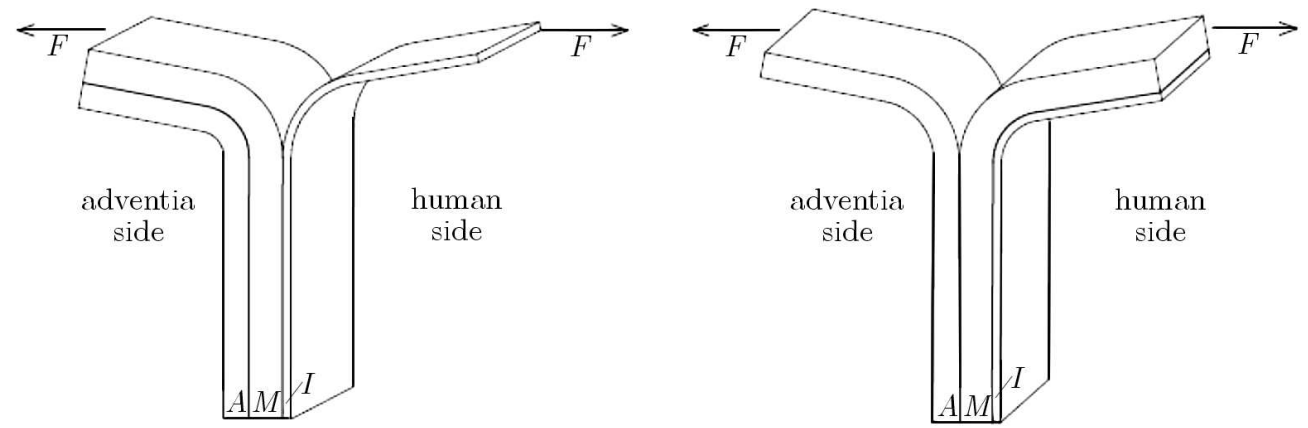

Fig. 3. Schematic illustration of the peeling test: (a) for interface No. 1 and (b) for interface No. 2. The letters $A, M$, and $I$ mark layers of the vessel wall: $A$-adventitia, $M$ - media, and $I$ - intima

\subsection{Statistical analysis}

A statistical analysis was performed using the nonparametric Wilcoxon test (Statistica 10.0, StatSoft). This test was performed at a statistically significant level of $p=0.05$. The values of mechanical parameters are presented as median values $(M e)$. Additionally, in order to compare these results with the data presented in the literature, the values of mechanical parameters are also presented as arithmetic mean with standard deviation $\left(X_{\text {mean }} \pm S D\right)$.

\section{Results}

The peeling test causes slow and controlled delamination propagation of human arteries. Figure 4 shows an example of the force per width $[\mathrm{mN} / \mathrm{mm}]$ vs the dissection path curve $[\mathrm{mm}]$ (Fig. 5) determined on the basis of the peeling test. All curves obtained for both circumferential and

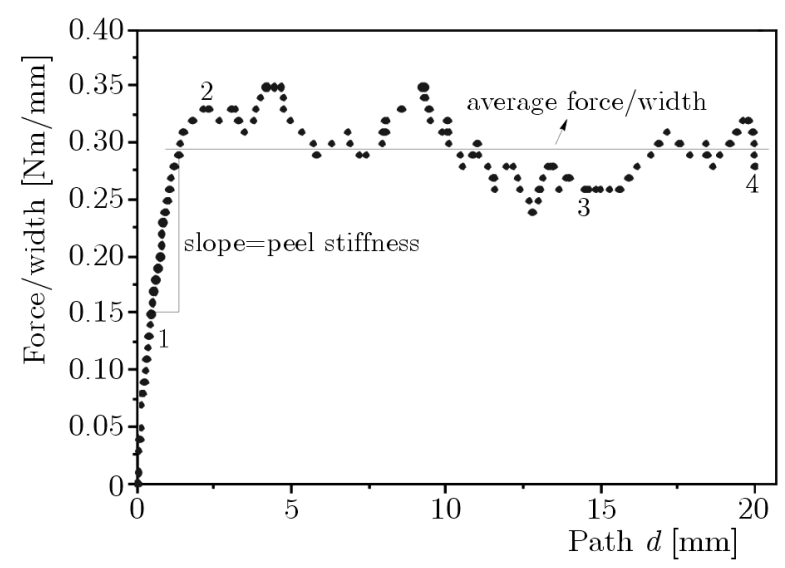

Fig. 4. The force/width vs the path curve obtained on the basis of the peeling test for interface No. 2 in the axial direction with the marked method of determining the mechanical properties, i.e. the stiffness $(k)$ and the average value of the force in relation to the width of the specimen $(F / W)$ during dissection 

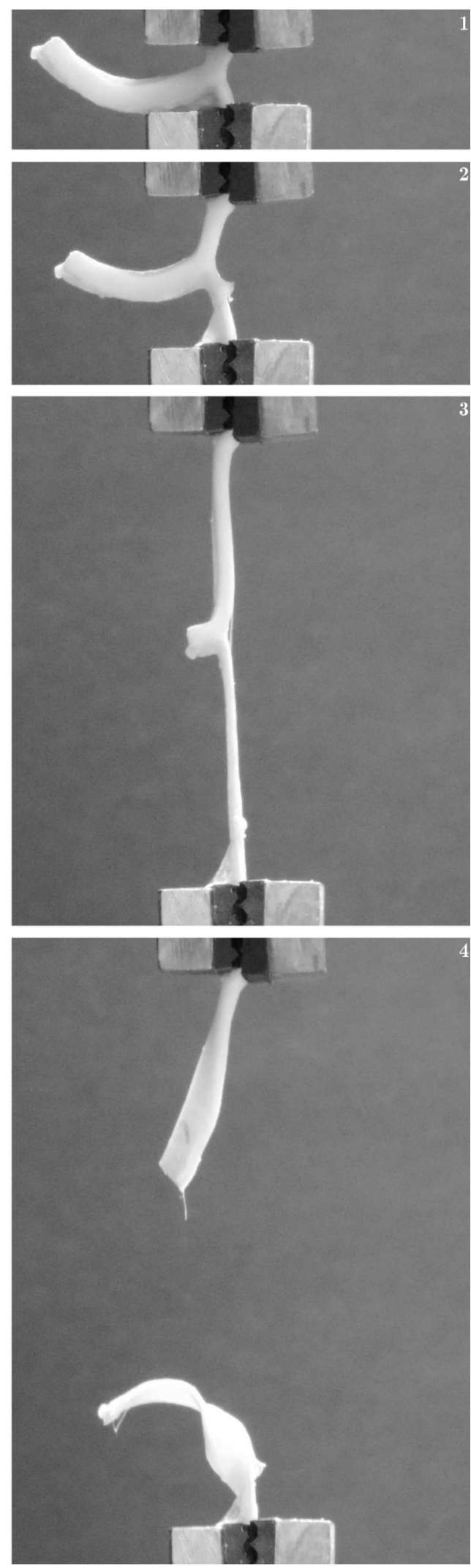

Fig. 5. Axial specimen of the arterial wall (interface No. 2) tested in the T-peel test configuration; four images $(1,2,3,4)$ show progression of the peel test until complete separation of the tissue (4). Examples of where each image (1-4) could fall on the force/width vs path curve (Fig. 4) are indicated by numbers $1,2,3$, and 4 
axial specimens are characterized by a jagged plateau region. Those curves were divided into two stages. Stage I is the linear part of the curve, which is characterized by high dynamics of the change in the force in relation to the change in displacement. This part was used to determine stiffness (k) (Gregory et al., 2012) of the interfaces between the layers of the human thoracic aortic wall (Fig. 4). During stage I, the strength of the tested interface is exceeded, and then stage II begins. At this stage, the aortic wall dissection is propagated as a result of tearing of individual collagen and elastin fibres, as evidenced by local increases and decreases in the value of the force (Fig. 4). For stage II, the average force value was determined, obtained during the process of aortic wall dissection in relation to the initial width of the specimen $(F / W)$ (Fig. 2), which was motivated by assuming ideal rectangular geometries and homogeneous mechanical properties of each specimen (Sommer et al., 2008).

The mechanical properties of both soft tissues (Maksymowicz et al., 2011; Pezowicz, 2010; Żak et al., 2011) and hard tissues (Nikodem, 2012) are analysed in the literature on the basis of the power criterion (Żak, 2014). In the presented work, the process of dissection of thoracic arterial wall has been described as cracking of the three-layer laminate using the energy balance, as previously proposed by Sommer et al. (2008) and Tong et al. (2011)

$$
W_{C}=\frac{W_{C}^{\text {ext }}-W_{C}^{\text {stor }}}{L_{C}} \quad W_{A}=\frac{W_{A}^{\text {ext }}-W_{A}^{\text {stor }}}{L_{A}}
$$

where $C, A$ are indications of the direction: $C$ - circumferential, $A$-axial, $L$ is reference length of the specimen, $W^{e x t}$ - external energy supplied to the system, $W^{\text {stor }}$ - energy stored in the system.

External energy is defined as follows (Tong et al., 2011)

$$
W_{C}^{e x t}=2.0 F_{C} l_{C} \quad W_{A}^{e x t}=2.0 F_{A} l_{A}
$$

where: $F$ - ratio of the force recorded during the test to the specimen width, $l$ - length of the specimen prior to dissection (before mounting in the grips of the material testing machine).

The energy stored in the system was calculated as follows (Tong et al., 2011)

$$
W_{C}^{\text {stor }}=F_{C}\left(l_{C}-L_{C}\right) \quad W_{A}^{\text {stor }}=F_{A}\left(l_{A}-L_{A}\right)
$$

where: $F$ - ratio of the force recorded during the test to the specimen width, $L$ - length of the specimen after dissection (after mounting in the grips of the material testing machine).

Table 2. The value $(M e)$ of dissipated energy $(W)$ during dissection of the human thoracic arterial wall

\begin{tabular}{|c|c|}
\hline Type of interface & $W\left[\mathrm{~mJ} / \mathrm{cm}^{2}\right]$ \\
\hline \hline \multicolumn{2}{|c|}{ Circumferential direction } \\
\hline interface 1 & 4.9 \\
\hline interface 2 & 4.5 \\
\hline \multicolumn{2}{|c|}{ Axial direction } \\
\hline interface 1 & 7.2 \\
\hline interface 2 & 6.2 \\
\hline
\end{tabular}

Both the stiffness values as well as force/width are significantly higher for axial specimens. Regardless of the analysed direction, statistically significant differences have been found between the stiffness of the tested interfaces. For axial specimens, the stiffness values $M e$ are, respectively, $k=0.17 \mathrm{~N} / \mathrm{mm}$ and $k=0.11 \mathrm{~N} / \mathrm{mm}$. The energy dissipated during propagation of aortic wall dissection is higher for axial specimens in the case of both interface No. 1 and interface No. 2 (6). These values $(M e)$ are, respectively, as follows: $W=7.2 \mathrm{~mJ} / \mathrm{cm}^{2}$ and $W=6.2 \mathrm{~mJ} / \mathrm{cm}^{2}$ $\left(X_{\text {mean }} \pm S D: 7.6 \pm 1.7 \mathrm{~mJ} / \mathrm{cm}^{2}\right.$ and $\left.4.7 \pm 0.9 \mathrm{~mJ} / \mathrm{cm}^{2}\right)$. In the case of both axial and circumferential specimens, higher energy values were obtained for interface No. 1 (Fig. 6). 


\section{Discussion}

The present study has been conducted to explain delamination of the human thoracic artery with stage II atherosclerotic lesions according to Stary $(2000,2004)$. The study included a peeling test which was used to determine the force per width, stiffness, and dissection energy of the adventitia-media+intima interface and the adventitia+media-intima interface in the circumferential and axial directions. The obtained results were used to characterise the resistance of the thoracic aortic wall as a three-layer laminate to propagation of delamination. The problem of delamination of the vessel wall is a new issue in the literature and only two papers (Sommer et al., 2008; Tong et al., 2011) attempted to describe the mechanism of this process.

Sommer et al. (2008) and Tong et al. (2011) determined the force per width and the energy dissipated during the process of delamination of the media of normal vessels: human abdominal artery and human carotid bifurcation. The analysis was conducted in the circumferential and axial directions relative to the long axis of the vessel. The results obtained by Sommer et al. (2008) and Tong et al. (2011) are higher for axial specimens. In this work, the obtained values of energy and force per width are comparable to the values obtained by Sommer et al. (2008) and Tong et al. (2011). In each of the analysed cases, the adventitia-media+intima interface is characterised by higher values of the mechanical parameters (Table 3). These differences are statistically significant $(p=0.05)$. None of the previous papers have analysed the values of stiffness of the interfaces between vessel wall layers, which in this paper, as in the case of other parameters, is higher for longitudinal specimens. In each of the analysed directions, statistically significant differences have been found between the stiffness of interface No. 1 and interface No. 2, with the higher values of this parameter obtained in the second case (Table 3).

Table 3. Comparison of the average values of mechanical parameters: energy $(W)$, force per width $(F / W)$, and stiffness $(k)$ obtained by Sommer et al. (2008) and Tong et al. (2011) and the results of own research in two directions: axial $(A)$ and circumferential $(C)$

\begin{tabular}{|c|c|c|c|c|c|c|c|}
\hline $\begin{array}{c}\text { Type } \\
\text { of } \\
\text { interface }\end{array}$ & \multicolumn{2}{|c|}{$\begin{array}{c}W \\
{\left[\mathrm{~mJ} / \mathrm{cm}^{2}\right]}\end{array}$} & \multicolumn{2}{c|}{$\begin{array}{c}F / W \\
{[\mathrm{mN} / \mathrm{mm}]}\end{array}$} & \multirow{2}{*}{ Source } \\
\cline { 2 - 7 } & $A$ & $C$ & $A$ & $C$ & $A$ & $C$ & \\
\hline \hline \multirow{2}{*}{$A-M I$} & $6.5 \pm 2.7$ & $5.0 \pm 1.0$ & $29.1 \pm 12.2$ & $22.7 \pm 4.5$ & - & - & {$[25]$} \\
\cline { 2 - 8 } & $7.6 \pm 1.7$ & $5.6 \pm 0.9$ & $32.4 \pm 6.5$ & $24.5 \pm 7.5$ & $0.20 \pm 0.08$ & $0.13 \pm 0.05$ & own research \\
\hline \multirow{2}{*}{$A M-I$} & $5.2 \pm 3.1$ & $3.6 \pm 0.7$ & $23.3 \pm 13.8$ & $16.4 \pm 3.3$ & - & - & {$[25]$} \\
\cline { 2 - 7 } & $4.7 \pm 0.9$ & $4.1 \pm 1.0$ & $34.2 \pm 3.5$ & $26.5 \pm 6.7$ & $0.19 \pm 0.07$ & $0.013 \pm 0.07$ & own research \\
\hline$M$ & $7.6 \pm 2.7$ & $5.1 \pm 0.6$ & $34.8 \pm 15.5$ & $22.9 \pm 2.9$ & - & - & {$[18]$} \\
\hline
\end{tabular}

[18] - Sommer et al. (2008), [25] - Tong et al. (2011)

Studies of the mechanical properties of the interfaces between vessel wall layers, conducted by Sommer et al. (2008) and Tong et al. (2011), concerned normal vessels, while dissection of the vessel wall is related to the occurrence of lesions, e.g. atherosclerosis. Hypothetically, structural remodelling of the aortic wall, which occurs even in the early stages of the disease, changes the mechanical parameters of vessel wall layers (Holzapfel, 2008; Teng et al., 2009; Weisbecker et al., 2012) and affects the adhesion between them. Consequently, atherosclerosis is believed to increase the risk of dissection. In the present study, mechanical parameters were designated for blood vessels in stage II of the development of atherosclerosis. The obtained results are similar to the distribution characteristics and values of the results obtained for normal vessels (Sommer et al., 2008; Tong et al., 2011).

On this basis, it can be concluded that the early stage of atherosclerosis does not affect the mechanical parameters of the interfaces between the layers of the aortic wall and, consequently, does not affect vessel the wall resistance to delamination. The test results show that the 
(a)

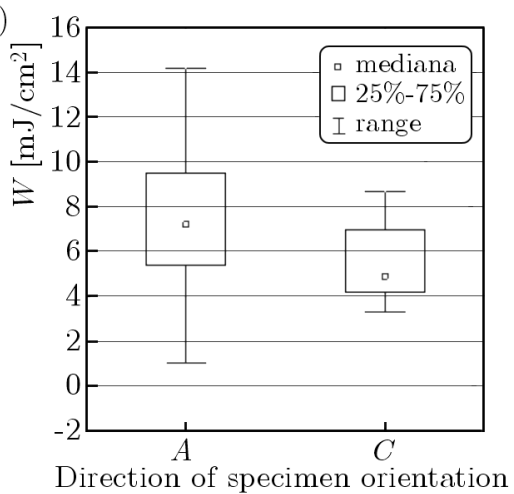

(b)

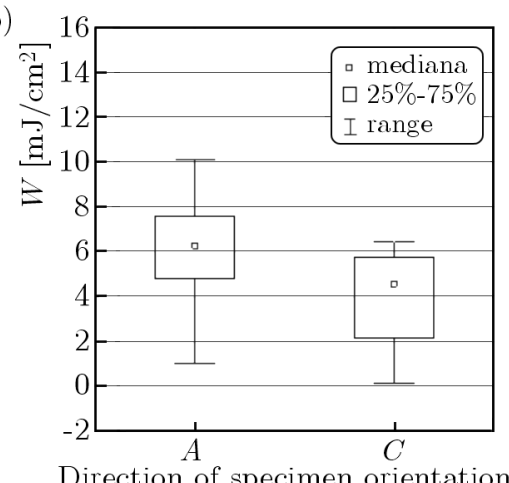

Fig. 6 . The energy values obtained during dissection of the human thoracic aortic wall depending on the peeling test direction ( $A$ - axial direction, $C$ - circumferential direction): (a) for interface 1 and

(b) for interface 2

mechanical and dissection properties of the interfaces between the layers of the human thoracic aortic wall depend on the specimen orientation. It is worth noting, however, that in contrast to the mechanical properties of the vessel wall, lower values of those parameters were obtained for circumferential specimens. This study demonstrated that lower dissection energy is required in the circumferential peeling test compared with the axial peeling tests, which may be related to the multiphase structure of the vessel wall and alignment of collagen and elastin fibres and smooth muscle cells. Dissection disseminates in the circumferential direction along elastic laminae, while in the axial direction it crosses elastic layers and the external or internal elastic laminae. In addition, circumferential alignment of smooth muscle cells and collagen and elastin fibres in the media of the vessel wall leads to stronger resistance to dissection during the peeling test in the axial direction (Tong et al., 2011). Circumferential specimens are also characterized by a lower stiffness value. Regardless of the analysed direction, lower dissection energy is needed to propagate dissection of the adventitia+media-intima interface. The energy values obtained for the interface between these layers are lower by $38 \%$ (axial specimens) and $27 \%$ (circumferential specimens) compared with the dissection energy obtained for the adventitia-media+intima interface. This shows that the adventitia+media-intima interface is most prone to delamination. In the case of stiffness, regardless of the analysed direction, both interfaces have the same value of the parameter.

In the experiment conducted in the study, the process of thoracic aortic wall dissection progressed in a controlled manner, while clinical circumstances of the dissection were more diverse. As a result, the obtained values of the mechanical and dissection properties might not be representative for in vivo aortic dissection. Despite this, in the opinion of the author, the obtained results of the experiments may be useful for the estimation of the response to the artery dissection and may improve clinical assessment, diagnosis, balloon angioplasty, and cardiovascular medicine.

\section{Acknowledgement}

This work has been part of the project "WROVASC - Integrated Cardiovascular Centre", co-financed by the European Regional Development Fund within Innovative Economy Operational Programme 2007-2013.

\section{References}

1. Becker A, Epple M., Muller K.M., Schmitz J., 2004, A comparative study of clinically well-characterized human atherosclerotic plaques with histological, chemical and ultrastructural methods, Journal of Inorganic Biochemistry, 98, 12, 2032-2038 
2. Carmo M., Colombo L., Bruno A., Corsi F.R., Roncoroni L., Cuttin M.S., Radice F., Mussini E., Settembrini P.G., 2002, Alteration of elastin, collagen and their cross-links in abdominal aortic aneurysm, European Journal of Vascular and Endovascular Surgery, 23, 6, 543-549

3. Gąsior-Gøogowska M., Komorowska M., Hanuza J., Ptak M., Kobielarz M., 2011, Structural alteration of collagen fibers-spectroscopic and mechanical studies, Acta of Bioengineering and Biomechanics, 12, 4, 55-62

4. Gregory D.E., Bae W.C., Sah R.L., Masuda K., 2012, Anular delamination strength of human lumbar intervertebral disc, European Spine Journal, 21, 1716-1723

5. Holzapfel G.A., 2008a, Artierial Tissue in Health and Disease: Experimental Data, CollagenBased Modeling and Simulation, Including Aortic Dissection, Lecture Notes, Graz University of Technology, Austria,

6. Holzapfel G.A., 2008b, Chapter no. 11, Collagen in arterial walls: biomechanical aspects, [In:] Collagen Structure and Mechanics, Peter Fratzl (Edit.), LLC, 285-324

7. Holzapfel G.A., Gasser Th.C., Ogden R.W., 2004, Comparison of a multi-layer structural model for arterial walls with a Fung-type model for arterial aalls with a Fung-type model and issues of material stability, Journal of Biomechanical Engineering, 126, 2, 264-275

8. Holzapfel G.A, Ogden R.W., 2003, Biomechanics of Soft Tissue in Cardiovascular Systems, Springer Wien New York

9. Karimi A., Navidbakhsh M., Shojaei A., Faghini S., 2013, Measurement of the uniaxial mechanical properties of healthy and atherosclerotic human coronary arteries, Materials Science and Engineering, 33, 5, 2550-2554

10. Kobielarz M., Jankowski L., 2013, Experimental characterization of the mechanical properties of the abdominal aortic aneurysm wall under uniaxial tension, Journal of Theoretical and Applied Mechanics, 51, 4, 949-958

11. Kot M., Kobielarz M., Maksymowicz K., 2011, Assessment of mechanical properties of arterial calcium deposition, Transactions of FAMENA, 35, 3, 49-56

12. Maksymowicz K., Kobielarz M., CzoŁgaŁa J., 2011, Potential indicators of the degree of abdominal aortic aneurysm development in rupture risk estimation, Advances in Clinical and Experimental Medicine, 20, 2, 221-225

13. Nikodem A., 2012, Correlations between structural and mechanical properties of human trabecular femur bone, Acta of Bioengineering and Biomechanics, 14, 2, 37-46

14. Pezowicz C., 2010, Analysis of selected mechanical properties of intervertebral disc annulus fibrosus in macro and microscopic scale, Journal of Theoretical and Applied Mechanics, 48, 4, 917-932

15. Schriefl A.J., Zeindlinger G., Pierce D.M., 2012, Determination of the layer-specific distributed collagen fibre orientations in human thoracic and abdominal aortas and common iliac arteries, Journal of the Royal Society Interface, 9, 1275-1286

16. Schulze-Bauer C., Regitinig P., Holzapfel G.A., 2001, Mechanics of the human femoral adventitia including high-pressure response, American Journal of Physiology, 282, 6, H2427-2440

17. Shekhonin B.V., Domogatsky S.P., Muzykantov V.R., Idelson G.L., Rukosuev V.S., 1985, Distribution of type I, III, IV and V collagen in normal and atherosclerotic human arterial wall: Immunomorphological characteristics, Coll. Relat. Res., 5, 355-368

18. Sommer G., Gasser T.C., Regitnig P., Auer M., Holzapfel G.A., 2008, Dissection properties of the human aortic media: an experimental study, Journal of Biomechanical Engineering, 130, 2, 1-12

19. Sommer G., Regitnig P., Kltringer L., Holzapfel G.A., 2010, Biaxial mechanical properties of intact and layer-dissected human carotid arteries at physiological and supraphysiological loadings, Heart and Circulatory Physiology - American Journal of Physiology, 298, 3, H898-H912 
20. Stary H.C., 2000a, Natural history of calcium deposits in atherosclerosis progression and regression, Cardiology Journal, 89, (Suppl 2): II/28-II/35

21. Stary H.C., 2000b, Natural history and histological classification of atherosclerotic lesions: an update, Arteriosclerosis, Thrombosis and Vascular Biology, 20, 1177-1178

22. Stary H.C., 2004, Atlas of Atherosclerosis Progression and Regression, CD-ROM, 2nd Edition

23. Szpakowski E., Kotliński K., Janaszek-Sitkowska H., 2006, Acute aortic dissection type A with concomitant myocardial infarction, Folia Cardiologica, 13, 1, 68-72

24. Teng Z., Tang D., Zheng J., Woodard P.K., Hoffman A.H., 2009, An experimental study on the ultimate strength of the adventitia and media of human atherosclerotic carotid arteries in circumferential and axial direction, Journal of Biomechanics, 42, 15, 2535-2539

25. Tong J., Sommer G., Regitnig P., Holzapfel G.A., 2011, Dissection properties and mechanical strength of tissue components in human carotid bifurcation, Annals of Biomedical Engineering, 39, 6, 1703-1719

26. Vorp D.A., Raghavan M.L., Muluk S.C., Makaroun M.S., Steed D.L., Shapiro R., WeBster M.W., 1996, Wall strength and stiffness of aneurysmal and nonaneurysmal aorta, Annals of the New York Academy of Science, 18, 800, 274-276

27. Vorp D.A., Schiro B.J., Ehrlich M.P., Juvonen T.S., Ergin M.A., Griffith B.P., 2003, Effect of aneurysm on the tensile strength and biomechanical behavior of the ascending thoracic aorta, The Annals of Thoracic Surgery, 75, 4, 1210-1214

28. Weisbecker H., Pierce D.M., Regiting P., Holzapfel G.A., 2012, Layer - specific damage experiments and modeling of human thoracic and abdominal aortas with non-atherosclerotic intimal thickening, Journal of the Mechanical Behavior of Biomedical Materials, 12, 93-106

29. ŻAK M., 2014, Effect of support on mechanical properties of the intervertebral disc in long-term compression testing, Journal of Theoretical and Applied Mechanics, 52, 3, 677-686

30. Żak M., Kuropka P., Kobielarz M., Dudek A., Kaleta-Kuratewicz K., Szotek S., 2011, Determination of the mechanical properties of the skin of pig foetuses with respect to its structure, Acta of Bioengineering and Biomechanics, 13, 2, 37-43

Manuscript received December 23, 2014; accepted for print August 12, 2015 